\title{
The dynamic code HSR-dyna: an efficient tool for the assessment of vibrations induced by high speed rail lines
}

\author{
R. Mezeh ${ }^{1,2}$, M. Sadek ${ }^{1}$, F. Hage Chehade ${ }^{2}$, A. Arab ${ }^{3}$ \\ \& I. Shahrour ${ }^{1}$ \\ ${ }^{1}$ Laboratory of Civil Engineering and geo-Environment ( $\left.L G C g E\right)$, \\ Lille 1 University, France \\ ${ }^{2}$ Doctoral School of Science \& Technology, Lebanese University, \\ Lebanon \\ ${ }^{3}$ Laboratory of Materials Science and Environment, \\ University of Hassiba Ben BouAli, Algeria
}

\begin{abstract}
The main objective of this paper is to outline a novel simulation tool in the time domain, HSR-dyna, to predict the level of impact vibrations induced by the passage of high speed train HST over a railway track. Consequently, a vertical train-track interaction model which is widely presented in the literature was used to quantify the contact forces on the wheel-rail interface. The train with four wheelsets representing two bogies is a ten degree of freedom subsystem, the track is modelled as an infinite Euler-Bernoulli beam supported by a viscoelastic layer and the two subsystems are coupled together via the non-linear Hertz contact mechanism. A vast array of model permutations is computed to assess the effect of each input parameter on the response of the dynamic system, comprising the velocity of the HST and the severity of track irregularity.

Keywords: high speed train, railway response, finite element method, Hertz contact theory.
\end{abstract}

\section{Introduction}

Railway noise and the induced vibrations transmitted into the ground are considered a major problem, in view of their influence on the conformity of the 
neighbouring population. Several authors have been interested in dealing with a problem of interaction between train and track to quantify the induced noise level and then compare it with the acceptable level that is mentioned in the technical regulation. In this context, in France the Noise Act 1992 imposes the acceptable noise level limits for residents; at the European scale, the maximum noise levels are limited at source by the SPI.

Under relevance in the analysis and design of railways, the dynamic response of beams resting on elastic foundation and subjected to moving loads has been widely studied. In this context, many analytical methods have been proposed by many researchers. Timoshenko et al. [1] solved the problem of free vibration of beams on elastic foundation. Ono and Yamada [2] have developed analytical formulas designed to estimate the amplitude of the vibrations generated by rail through the geometrical defects at the wheel or rail. However, for general problems with complex issues, numerical methods must be used.

The finite element method is a well-established numerical method to solve complex problems including the case of moving loads. Note that this method was applied for the first time to solve a moving load problem by Yoshida and Weaver [3]. It has since been used by many other researchers. Yokoyama [4] developed a finite element procedure for determining the vibration characteristics of a uniform Timoshenko beam-column on elastic foundation. Thambiratnam and Zhuge [5] used the Newmark integration scheme to find the influence of the load velocity and the foundation stiffness on the dynamic amplification of a beam on elastic support.

In dealing with moving load problems, the classical FEM encounters difficulty when the moving load travels in high speed or/and the dynamic excitation has a small wavelength; sometimes these difficulties can be overcome by a refinement of the mesh, but at the expense of significant increase in computational time. In an attempt to overcome the complication encountered by the FEM, Krenk et al. [6] proposed the use of the FEM in convected coordinates to obtain the response of an elastic half-space subjected to a moving load. Koh et al. [7] adopted the idea of convected coordinates for solving train-track problems, and named the numerical algorithm as moving element method MEM. This method was subsequently applied to the analysis of moving loads on a viscoelastic half-space by Tran et al. [8].

In this paper, the convection problem including a discrete system with multiple contact points with an infinite Euler-Bernoulli beam resting on a viscoelastic layer (Kelvin foundation), simulating the interaction between the high speed train and the railway track, was treated by a new numerical approach. By adopting an appropriate time-space coupled mesh, the resulting numerical system was solved by an adaptive algorithm. The performance of this method has been tested for the excitation in the form of relative roughness between rail and wheel. A vast array of model permutations is computed to assess the effect of each input parameter on the response of the dynamic system, comprising the velocity of the HST and the severity of track irregularity. 


\section{Mathematical formulation}

The HSR system comprises of a train traversing over a rail beam in the positive $\mathrm{X}$-axis is arbitrary located along the beam, for convenience, its origin is taken such that the axis of the train is at $\mathrm{X}=0$ when $\mathrm{t}=0$. The constant velocity of the train is designed by $\mathrm{V}$. The rolling surface of the rail is assumed to have some imperfections resulting in the so-called track irregularity. The moving sprungmass model (as shown in Fig. 1), is employed to model the train. In this study, the effect of shear deformation and rotational inertia of the cross-section of the beam are neglected (Euler-Bernoulli theory). The Winkler hypothesis is taken into account which postulates that a foundation behaves like an infinite series of closely spaced, independent, linearly elastic, vertical springs. The damping of the material forming the foundation is accounted through linear viscous dampers continuously distributed beneath the Euler-Bernoulli beam.

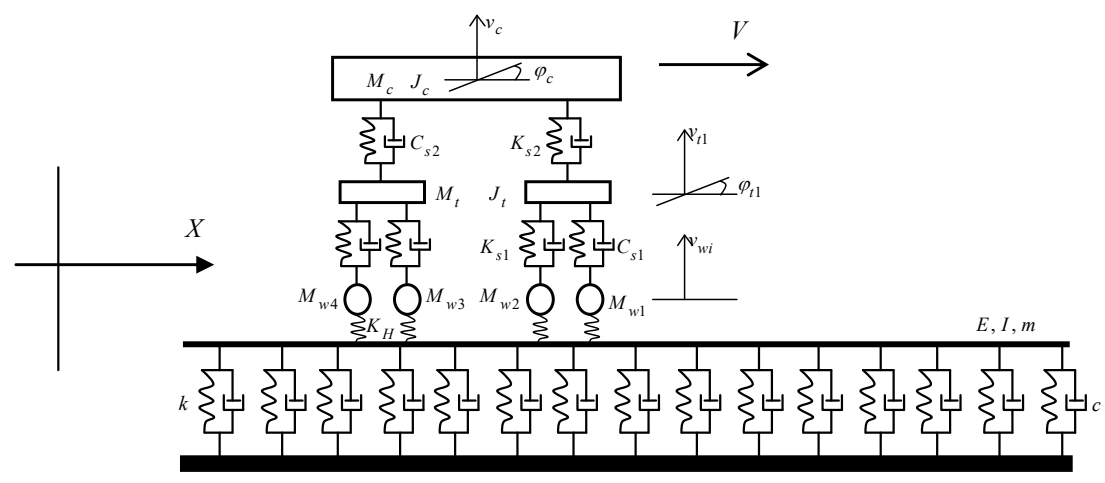

Figure 1: High speed train-railway track interaction model.

\subsection{HST-track dynamic coupling system}

Based on the theory of vehicle-track coupling dynamics and considering the highspeed vehicle and track as a system (see Fig. 1), a vertical dynamic analysis model for the high-speed train-track coupling system is developed. The following assumptions are made in the model.

- The vehicle and the railway track are considered to be symmetrical about the centerline of the track, and the longitudinal motion of the track has no effect in railway tangent motion; only half of the coupling system and only vertical vibrational behavior are considered in the simplified calculation.

- The full vehicle is represented by a rigid body model of a car body, two bogies, and four wheelsets connected to each other with springs and dampers.

- The vehicle system and the track system interact through the wheel/rail vertical force, which is approximated by Hertz contact theory. 
- The rail is considered as an infinite Euler beam that is supported discretely by linear viscoelastic layer; the sleepers are considered as a point mass that are supported by another viscoelastic layer, which simulate the connections between the sleepers and the ballast.

\subsubsection{Dynamic equation of vehicle}

The upper structure in the coupled system is represented by a railway vehicle with two layers of springs and dampers representing the primary and secondary suspension systems; in which the vertical movement and the pitch plane rotation for the vehicle body and bogies are considered.

The vehicle body of mass $\mathrm{M}_{\mathrm{c}}$ and moment of inertia $\mathrm{J}_{\mathrm{c}}$ is connected with two bogies of mass $\mathrm{M}_{t}$ and moment of inertia $\mathrm{J}_{\mathrm{t}}$ each, via the secondary suspension which is modeled as a spring $\mathrm{K}_{\mathrm{s} 2}$-damper $\mathrm{C}_{\mathrm{s} 2}$ unit. The interconnections between bogies and wheelsets of mass $\mathrm{M}_{\mathrm{w}}$ each are conducted via the primary suspension system $\mathrm{K}_{\mathrm{s1}}-\mathrm{C}_{\mathrm{s} 1}$. In this case the vehicle has ten degrees of freedom, seven vertical displacement and three plane rotations.

Defining the compete vehicle as a calculation element, the global displacement $\{\mathrm{a}\}_{\mathrm{u}}$ and load $\{\mathrm{Q}\}_{\mathrm{u}}$ vectors can be expressed as follows:

$$
\begin{array}{r}
\{a\}_{u}=\left\{\begin{array}{llllllllll}
v_{c} & \varphi_{c} & v_{t 1} & \varphi_{t 1} & v_{t 2} & \varphi_{t 2} & v_{w 1} & v_{w 2} & v_{w 3} & v_{w 4}
\end{array}\right\}^{T} \\
\{Q\}_{u}=\left\{\begin{array}{llllllllll}
-M_{c} g & 0 & -M_{t} g & 0 & -M_{t} g & 0 & P_{1} & P_{2} & P_{3} & P_{4}
\end{array}\right\}^{T}
\end{array}
$$

where

$$
P_{i}=-M_{w} g+f_{w i}
$$

$\mathrm{V}_{\mathrm{c}}$ and $\varphi_{\mathrm{c}}$ are the vertical displacement and pitch motion for the rigid body of the vehicle, $v_{t i}$ and $\varphi_{t i}$ are the vertical displacement and pitch motion for the ith bogie, $\mathrm{V}_{\mathrm{wi}}$ is the vertical displacement of the ith wheel and $f_{\mathrm{wi}}$ is the interaction force resulting from the ith wheel of the vehicle contact with the rail.

The dynamic equation of the vehicle in the coupled system can be given as:

$$
[M]_{u}\{\ddot{a}\}_{u}+[C]_{u}\{\dot{a}\}_{u}+[K]_{u}\{a\}_{u}=\{Q\}_{u}
$$

where $[\mathrm{M}]_{\mathrm{u}},[\mathrm{C}]_{\mathrm{u}}$ and $[\mathrm{K}]_{\mathrm{u}}$ are mass, damping and stiffness matrices for the upper structure, the dot denotes the derivation with respect to time.

\subsubsection{Dynamic equation of railway track}

The lower structure in the coupled system is the railway track where the rails are considered as beams of finite length resting on a continuous viscoelastic foundation comprising of vertical springs $\mathrm{k}$ and dashpots $\mathrm{c}$. The finite element method is employed to establish the dynamic equation of the railway track, where rails as beams with bending deformation are discretized as one-dimensional beam elements.

The finite element dynamic equation for the railway track can be written as:

$$
[M]_{l}\{\ddot{a}\}_{l}+[C]_{l}\{\dot{a}\}_{l}+[K]_{l}\{a\}_{l}=\{Q\}_{l}
$$


where

$$
\begin{gathered}
M_{l}=\sum\left[m \int_{0}^{L_{e}} H^{T} H d r\right] \\
C_{l}=\sum\left[c \int_{0}^{L_{e}} H^{T} H d r\right] \\
K_{l}=\sum\left[E I \int_{0}^{L_{e}}\left(\frac{\partial^{2} H}{\partial r^{2}}\right)^{T} \frac{\partial^{2} H}{\partial r^{2}} d r+k \int_{0}^{L_{e}} H^{T} H d r\right]
\end{gathered}
$$

$[\mathrm{M}]_{1},[\mathrm{C}]_{1}$ and $[\mathrm{K}]_{1}$ are the global mass, damping and stiffness matrices for the lower structure, which can be assembled by matrices of element mass, element damping and element stiffness. E, I, and $\mathrm{m}$ are Young's modulus, second moment of inertia, and mass per unit length of the rail beam respectively; $r$ is the local coordinate system of each finite element. For beam elements, it is common to use the shape function matrix $\mathrm{H}$ based on Hermitian cubic polynomials.

\subsubsection{High speed rail coupled system}

In this study, we consider the nonlinear contact theory of Hertz, for coupling the two discrete systems via the contact forces $f_{w i}$, which is calculated on the ith wheelrail interface as follows:

$$
f_{w i}= \begin{cases}K_{H}\left(\left|v_{w i}-\left(v_{x i}+\eta_{i}\right)\right|\right)^{3 / 2} & , \\ 0, & v_{w i}-\left(v_{x i}+\eta_{i}\right)<0 \\ & , \quad v_{w i}-\left(v_{x i}+\eta_{i}\right) \geq 0\end{cases}
$$

in which $v_{x i}$ and $v_{w i}$ denote the displacement of the rail and the ith wheel, respectively, and $\eta_{i}$ is the irregularity of the track vertical profile at the contact point with ith wheel. $\mathrm{K}_{\mathrm{H}}$ denotes the Hertizian spring constant. According to the recommendation by Nielsen and Abrahamsson [9], the track irregularity profile can be written in terms of a sinusoidal function as follows:

$$
y_{t}=a_{t} \sin \frac{2 \pi x}{\lambda_{t}}
$$

where $a_{t}$ and $\lambda_{t}$ denote the amplitude and wavelength of the track irregularity, respectively.

The global nodal displacement vector and load vector for the coupled system can be defined as:

$$
\begin{aligned}
\{a\} & =\left\{\begin{array}{ll}
a_{l} & a_{u}
\end{array}\right\}^{T} \\
\{Q\} & =\left\{\begin{array}{ll}
Q_{l} & Q_{u}
\end{array}\right\}^{T}
\end{aligned}
$$

The nonlinear dynamic equation of the vehicle-railway coupled system can be given as:

$$
[M]\{\ddot{a}\}+[C]\{\dot{a}\}+[K]\{a\}=\{Q\}
$$

$[\mathrm{M}],[\mathrm{C}]$ and $[\mathrm{K}]$ the global mass, damping and stiffness matrices of the coupled system. 


\subsection{Numerical algorithm}

In the numerical implementation, the vehicle and track coupled system are solved simultaneously with an iterative scheme. Coupling the vehicle system and the railway track can be realized through interaction forces between wheels and rail. The track vertical profile irregularity will be considered in calculating the interaction forces with the conventional Hertz formula.

\subsubsection{Step by step procedure in the time domain}

Assuming that in time step $(\mathrm{t}+\mathrm{dt})$ the $\mathrm{K}^{\text {th }}$ iteration has been done, and vectors of the displacement, velocity and acceleration at time step $(\mathrm{t})$ for the coupled system are obtained. Now let us consider the $(\mathrm{K}+1)^{\text {th }}$ iteration of the time step $(\mathrm{t}+\mathrm{dt})$; dynamic equations for the coupled structure will be solved by a combined Newmark-Newton-Raphson scheme. Fig. 2 shows the flowchart representing the numerical algorithm used in solving the system of nonlinear differential equations. Note that the matrices $[\mathrm{A}]$ and $[\mathrm{B}]$ are given as follows:

$$
A=\left[\begin{array}{l}
{\left[\frac{1}{\alpha d t^{2}} M+\frac{\delta}{\alpha d t} C+K\right]} \\
-\left[\frac{1}{\alpha d t^{2}} M+\frac{\delta}{\alpha d t} C\right] \\
-\left[\frac{1}{\alpha d t} M+\left(\frac{\delta}{\alpha}-1\right) C\right] \\
-\left[\begin{array}{ll}
\left.\left(\frac{1}{2 \alpha}-1\right) M+d t\left(\frac{\delta}{2 \alpha}-1\right) C\right]
\end{array}\right]
\end{array}\right.
$$

where $\alpha$ and $\delta$ are the Newmark's coefficients, $\mathrm{dt}$ is an infinitesimal time step and $\varepsilon$ is a specified tolerance. $\mathrm{J}$ is the Jacobian matrix.

To take into account the motion of the train along the track, it is proposed to solve the system by injecting after a specified time interval a new initial condition based on the interpolation Hermite polynomial. The general principle of this approach is to solve the problem on this small interval of time (number of time steps) by using the step by step procedure; this stage will be followed by a change in the configuration of the rail in terms of displacement, rotation and their first and second derivatives with respect to time.

\section{Numerical results}

In this section, the dynamic behavior of a passenger car of a French high speed train TGV in which its geometric configuration is presented by Connolly et al. [10] is studied. The train which travels at constant velocity $\mathrm{V}=200 \mathrm{Km} / \mathrm{h}$ is 
simulated using the moving sprung-mass model. The numerical model adopted in the study comprises of a truncated railway track of $60 \mathrm{~m}$ length discretized nonuniformly with elements ranging from a coarse $1 \mathrm{~m}$ to a more refined $0.5 \mathrm{~m}$ size. We adopted in this analysis the mechanical characteristics of the European highspeed rail which are presented by Mezeh et al. [11].

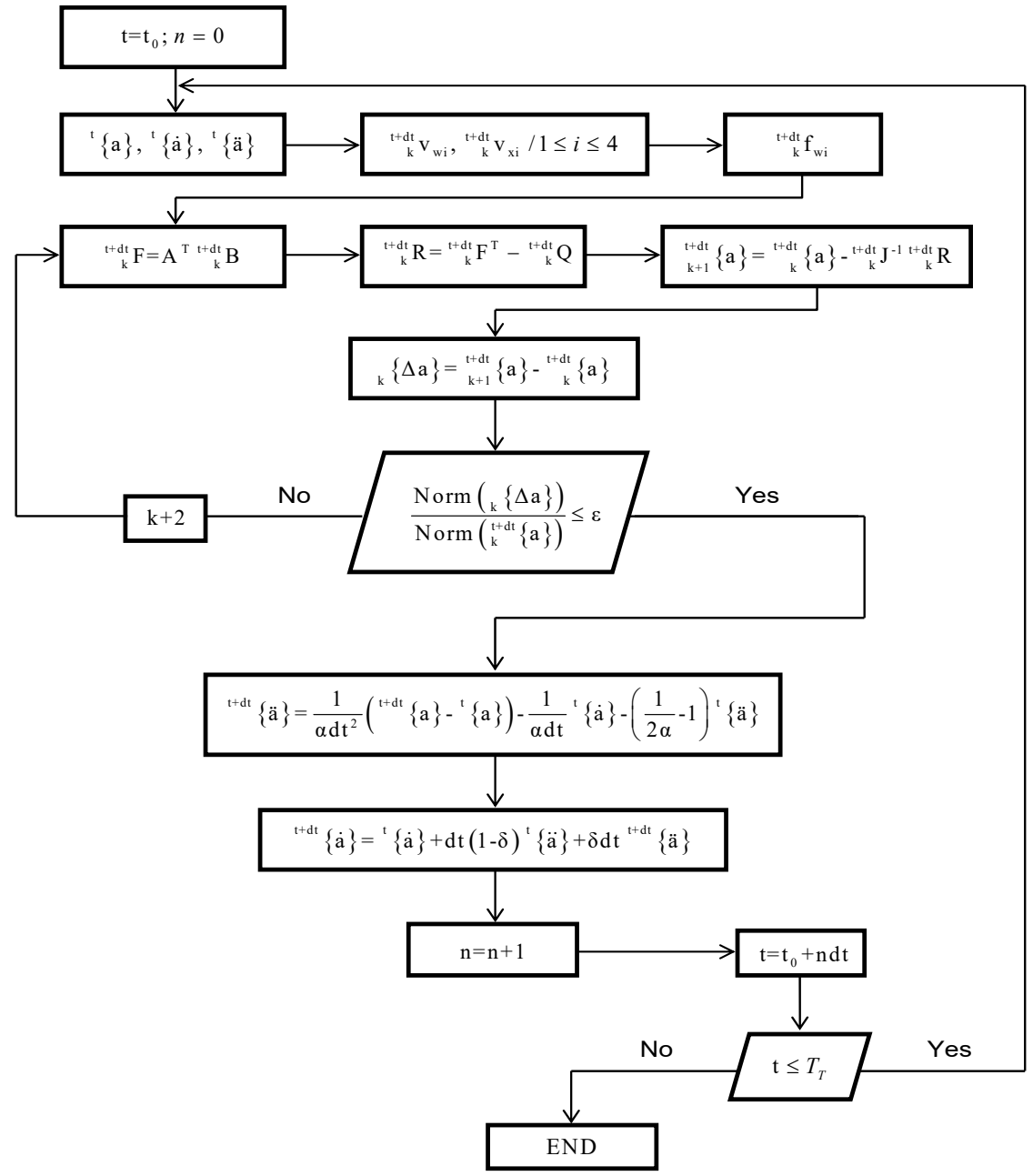

Figure 2: Flowchart of the adopted implicit-iterative scheme.

The rolling surface of the rail is supposed to be rough on a total length of $25 \mathrm{~m}$; the amplitude and wavelength of this harmonic irregularity are taken successively $\mathrm{a}_{\mathrm{t}}=0.5 \mathrm{~mm}$ and $\lambda_{\mathrm{t}}=1 \mathrm{~m}$. Figures $3(\mathrm{a})$ and $3(\mathrm{~b})$ show the dynamic response of the contact point on the mobile wheel-rail interface expressed on the time and 
frequency axis successively. Figure 4(a) shows the contact force $f_{w 1}$ diagram calculated according to eqn (9).

Fig. 4(b) shows the deformed rail, $0.55 \mathrm{sec}$ after the start of the TGV.

Table 1: Mechanical properties of the French TGV (Connolly et al. [10]).

\begin{tabular}{|c|c|}
\hline Mechanical properties & Value \\
\hline $\mathrm{M}_{\mathrm{c}}(\mathrm{Kg})$ & 24000 \\
\hline $\mathrm{M}_{\mathrm{t}}(\mathrm{Kg})$ & 3040 \\
\hline $\mathrm{M}_{\mathrm{w}}(\mathrm{Kg})$ & 2003 \\
\hline $\mathrm{K}_{\mathrm{s} 1}(\mathrm{MN} / \mathrm{m})$ & 1.4 \\
\hline $\mathrm{C}_{\mathrm{s} 1}(\mathrm{KNs} / \mathrm{m})$ & 120 \\
\hline $\mathrm{K}_{\mathrm{s} 2}(\mathrm{MN} / \mathrm{m})$ & 0.45 \\
\hline $\mathrm{C}_{\mathrm{s} 2}(\mathrm{KNs} / \mathrm{m})$ & 40 \\
\hline
\end{tabular}

(a)

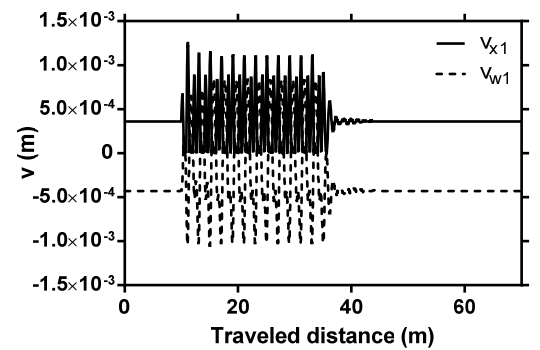

(b)

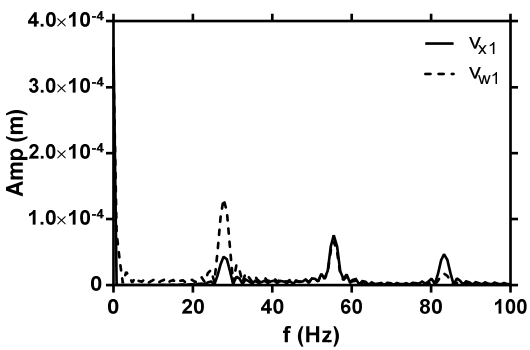

Figure 3: Dynamic response for the interaction model (a) on the time axis (b) on the frequency axis $\left(\mathrm{V}=200 \mathrm{Km} / \mathrm{h}, \mathrm{a}_{\mathrm{t}}=0.5 \mathrm{~mm}, \lambda_{\mathrm{t}}=1 \mathrm{~m}\right)$.

(a)

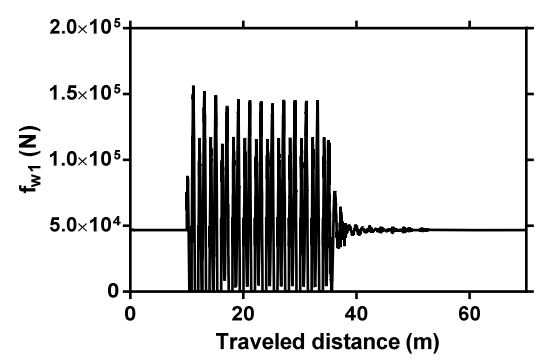

(b)

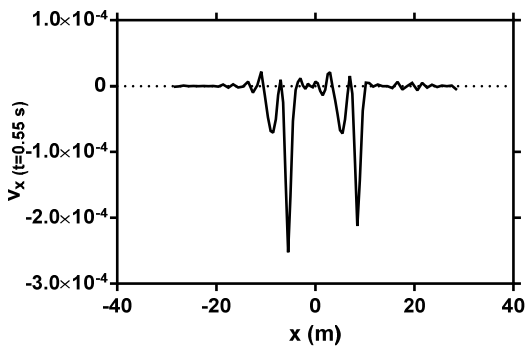

Figure 4: (a) Contact force fw1 (b) deformed rail at $\mathrm{t}=0.55 \mathrm{sec}(\mathrm{V}=200 \mathrm{Km} / \mathrm{h}$, $\mathrm{at}=0.5 \mathrm{~mm}, \lambda \mathrm{t}=1 \mathrm{~m})$. 


\subsection{Effect of track irregularity wavelength}

As the response of high-speed rail system strongly depends on the severity of track irregularity, it would be useful to investigate the effects of irregularity wavelengths and train speeds on the response of the HSR. The amplitude of all track irregularities considered in this investigation is taken to be $0.5 \mathrm{~mm}$. Figure 5 shows the dynamic amplification factor DAF in wheel-rail contact force against trainspeed for various track irregularity wavelength.

(a)

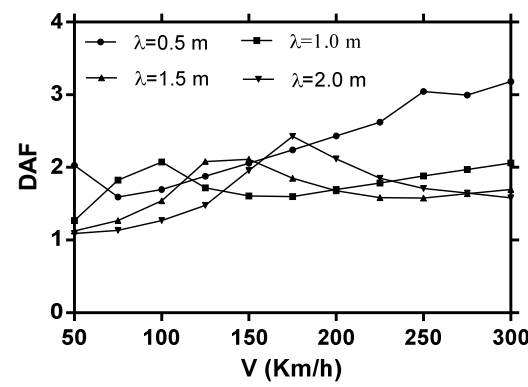

(b)

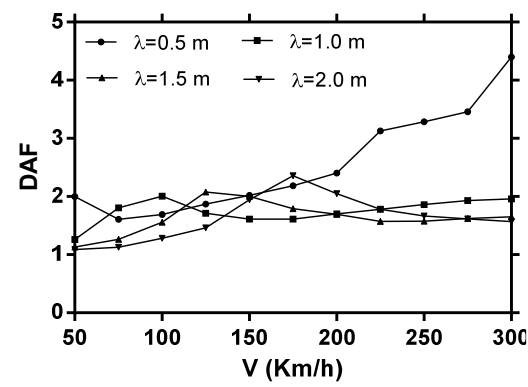

Figure 5: Dynamic amplification factor against train speed $\mathrm{V}$ for various track irregularity wavelength (a) front wheel (b) rear wheel.

The general trend of the curves is increasing with respect to the passing velocity, it can be seen the presence of a peak for each curve corresponds to a resonant speed.

\subsection{Effect of track irregularity amplitude}

The effect of train speed and track irregularity amplitude is investigated in this part. The wavelength of all track irregularities is taken to be $1.0 \mathrm{~m}$. Figure 6 shows the variation of the dynamic amplification factor (DAF) in wheel-rail contact force against train speed for various track irregularity amplitude.

(a)

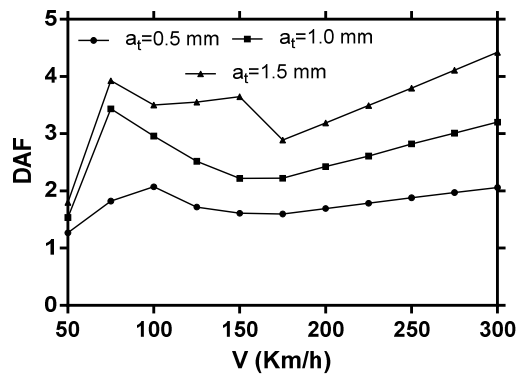

(b)

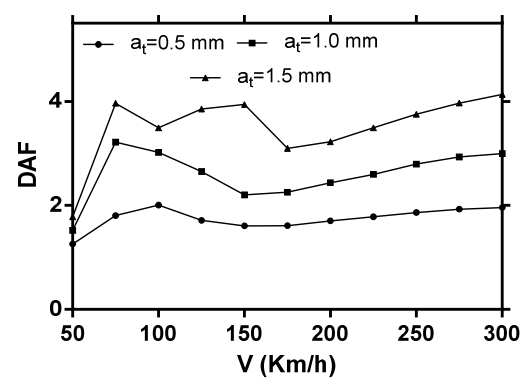

Figure 6: Dynamic amplification factor against train speed V for various track irregularity amplitude (a) front wheel (b) rear wheel. 
Figure 6 shows that the amplitude of the rail irregularity has no influence on the form of the obtained curves; its influence is limited to an increase in the DAF. For example, the DAF is subjected to a $43 \%$ relative increase when passing from $\mathrm{a}_{\mathrm{t}}=0.5 \mathrm{~mm}$ to $1 \mathrm{~mm}$ for $\mathrm{V}=200 \mathrm{Km} / \mathrm{h}$.

\section{Conclusion}

This paper presented a numerical model for the simulation of the dynamic interactions between the high speed train and the railway track by an adaptive aspect of the finite element method. The calculation model adopts the nonlinear Hertz contact theory to calculate the mutual interactions at the wheel-rail interface. It has demonstrated that the model proposed in the paper is an efficient research tool to investigate the dynamic effects caused by rail defect, which are damaging to the vehicle and track components. The relationship between the vehicle components, namely the unsprung mass and the dynamic wheel rail load, can be quantified using the established model.

\section{References}

[1] Timoshenko, S., Young, D.H. \& Weaver, W., Vibration problems in engineering, New York: John Wiley; fourth edition, 1974.

[2] Ono, K. \& Yamada, M., Analysis of railway track vibration. Journal of sound and vibration, 130, pp. 269-297, 1989.

[3] Yoshida, D.M. \& Weaver, W., Finite element analysis of beams and plates with moving loads. Publication of international association for bridge and structural engineering, 31(1), pp. 179-195, 1971.

[4] Yokoyama, T., Vibrations of Timoshenko beam-columns on two-parameter elastic foundations. Earthquake engineering \& structural dynamics, vol. 20, $\mathrm{n}^{\circ} 4$, pp. 355-370, 1991.

[5] Thambiratnam, D. \& Zhuge, Y., Dynamic analysis of beams on an elastic foundation subjected to moving loads. Journal of sound and vibration, 198, pp. 149-169, 1996.

[6] Krenk, S., Kellezi, L., Nielson, S.R.K. \& Kirkegaard, P.H., Finite elements and transmitting boundary conditions for moving loads. Proc. of the $4^{\text {th }}$ European conference on structural dynamics- EURODYN'99, pp. 447-452, 1999.

[7] Koh, C.G., Chiew, G.H. \& Lim, C.C., A numerical method for moving load on continuum. Journal of sound and vibration, 300, pp. 126-138, 2007.

[8] Tran, M.T., Ang, K.K. \& Luong, V.H., Vertical dynamic response of nonuniform motion of high-speed rails. Journal of sound and vibration, 333, pp. 5427-5442, 2014.

[9] Nielsen, J.C.O. \& Abrahamsson, T.J.S., Coupling of physical and modal components for analysis of moving non-linear dynamic systems on general beam structures. International Journal for Numerical Methods in Enginneering, 33, pp. 1843-1859, 1992. 
[10] Connolly, D.P., Kouroussis, G., Woodward, P.K., Alves Costa, P., Verlinden, O. \& Forde, M.C., Field testing and analysis of high speed rail vibrations. Soil Dynamics and Earthquake Engineering, 67, pp. 102-118, 2014.

[11] Mezeh, R., Sadek, M., Hage Chehade, F., Abdelmassih, D., Makhoul, N. \& Younes, A., Vibration analysis of high-speed rail system using the moving element method. Proc. $5^{\text {th }}$ International Conference on Computational Methods in Structural Dynamics and Earthquake Engineering, vol. 2, pp. 2951-2960, 2015. 